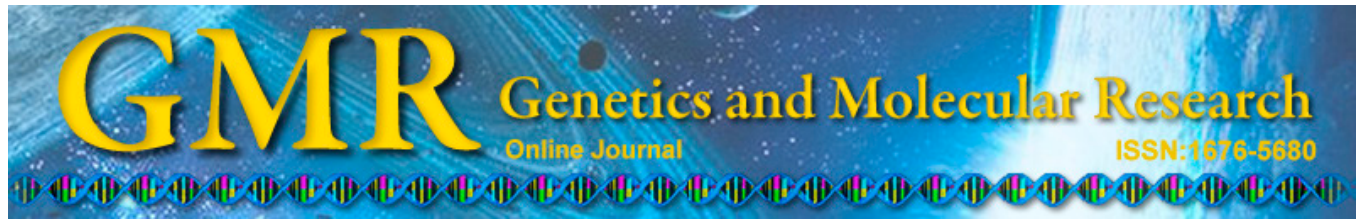

\title{
Molecular identity of ramie germplasms using simple sequence repeat markers
}

\author{
M.B. Luan ${ }^{1 *}$, B.F. Chen ${ }^{2 *}$, Z.Z. Zou ${ }^{3}$, J.J. Zhu ${ }^{1}$, X.F. Wang ${ }^{1}$, Y. Xu ${ }^{1}$, \\ Z.M. Sun ${ }^{1}$ and J.H. Chen ${ }^{1}$ \\ ${ }^{1}$ Institute of Bast Fiber Crops, Chinese Academy of Agricultural Sciences/ \\ Key Laboratory of Stem-Fiber Biomass and Engineering Microbiology, \\ Ministry of Agriculture, Changsha, China \\ ${ }^{2}$ Department of Medical Biology, North Sichuan Medical College, \\ Sichuan Nanchong, China \\ ${ }^{3}$ Yiyang Medical School, Yiyang, China \\ *These authors contributed equally to this study. \\ Corresponding author: J.H. Chen \\ E-mail: luanmingbao2002@126.com
}

Genet. Mol. Res. 14 (1): 2302-2311 (2015)

Received April 7, 2014

Accepted October 15, 2014

Published March 27, 2015

DOI http://dx.doi.org/10.4238/2015.March.27.15

\begin{abstract}
DNA identity is highly effective and efficient for distinguishing crop varieties regardless of their phenotypic similarities. To establish DNA identity in ramie, 21 simple sequence repeat primers were amplified in 108 accessions of domestic and exotic ramie germplasms. Sixty polymorphic bands were obtained, with an average of 2.9 bands per locus and 2-8 band types per primer locus (average of 5.19 band types). The Simpson's diversity index of the 21 simple sequence repeat loci ranged from 0.158 to 0.808 with an average of 0.612 . There was large difference in the specific index in the germplasm tested, from 44.082 to 218.163 , with an average of 83.620. Based on allele band type, 8 primer pairs were selected for DNA fingerprinting of the 108 genotypes. The combination of the 8 primer pairs were found to be very effective for distinguishing these genotypes, indicating that they can be used in the molecular DNA identity of ramie.
\end{abstract}

Key words: Molecular identity; Ramie; Simple sequence repeat 


\section{INTRODUCTION}

Ramie (Boehmeria nivea L.), also called Chinese grass, is principally used for fabric production and is one of the oldest fiber crops cultivated in China; this crop has been cultivated for more than 6000 years. It is a popular perennial plant and native to eastern Asia (Xiong, 2008). The bark of the vegetative stalks is the source of bast fiber. A series of ramie industry products have become popular in domestic markets, including shirts, underwear, and socks (Liu, 2002). In previous studies of ramie, various applications of ramie were established, with the exception of the fiber industry. Ramie is also cultivated as fodder crop in the southern regions of China because of its excellent nutritional value, containing $22 \%$ crude protein, $18 \%$ crude fiber, $1 \%$ Lys, $4 \% \mathrm{Ca}^{2+}$, and other nutrient elements needed by livestock regardless of the fresh leaf or its processing into dry power or silage (Yu et al., 2007; Xiong et al., 2010). Recently, ramie has become an optimal crop for conserving soil and water in the Yangtze River valley because of its abundant roots and luxuriant foliage (Tu and Chen, 2007).

In the ramie germplasm collection process, it is necessary to conduct cultivar identification for breeding selection and germplasm reservation. Traditionally, ramie germplasms are identified based on morphology, but this is laborious. Morphological traits must be obtained through multi-years and multi-sites, which is time consuming and costly. Additionally, the differences between morphological traits among germplasms are sometimes difficult to identify.

Molecular markers have been widely used to characterize ramie germplasms. For instance, random amplified polymorphic DNA (RAPD) (Jie et al., 1999; Guo et al., 2001; Meng et al., 2010), simple sequence repeats (SSR) (Zhou et al., 2004; Zou et al., 2012; Guo et al., 2013), inter-simple sequence repeat (ISSR) (Liu et al., 2006; Hou et al., 2006; Ding et al., 2008), sequence-related amplified polymorphism (SRAP) (Liu et al., 2008; Wen et al., 2011; Zou et al., 2012), restriction site amplification polymorphism (Zou et al., 2012), and random amplified microsatellite polymorphism (Zhou et al., 2004) were developed and shown to be rapid, accurate, and economical in ramie germplasm research. Moreover, several reports have illustrated the advantage of using molecular ID in crop species. Zheng et al. (2010) established 51 kenaf germplasms using of ISSR and RAPD markers. Liu et al. (2013) established 127 kenaf germplasms using SRAP markers. Wang et al. (2010) first distinguished 42 germplasms of ramie using 7 ISSR markers, and developed the first molecular ID of ramie. However, this plant shows poor stability and it is complex to record molecular ID because of the 16-digit code in the ID constructed using ISSR markers. Comparing with ISSR and other molecular markers, SSRs are highly polymorphic, informative, codominant, technically simple, and reproducible, and have become commonly used for constructing the molecular ID in the crop species (Fang et al., 2001). Some species have been evaluated by molecular ID using SSR markers. Gao et al. (2009) established 83 soybean germplasm molecular IDs using 9 SSR markers. Yang et al. (2010) established 36 tea germplasm molecular IDs using 17 SSR markers. Chen et al. (2011) established 202 peach germplasm molecular IDs using 8 SSR markers. Wang et al. (2011) established 142 sweet sorghum variety molecular IDs using 11 SSR markers. Zhang et al. (2014) established 20 pear variety molecular IDs using 2 SSR markers. However, there have been no reports related to molecular ID using SSR markers in ramie.

Therefore, the objective of this study was to construct molecular IDs of the 108 ramie germplasms using SSR markers. 


\section{MATERIAL AND METHODS}

\section{Materials and DNA isolation}

A total of 108 ramie accessions (Table 1) growing in a national ramie germplasm nursery affiliated with the Institute of Bast Fiber Crops, CAAS, China, were used in this study. DNA was isolated from young leaves collected from each ramie accession using the DNeasy plant mini prep kit (Qiagen, Hilden, Germany).

\begin{tabular}{|c|c|c|c|c|c|}
\hline Code & Entry & Origin & Code & Entry & Origin \\
\hline 1 & Wayaozhuma & Guangxi & 55 & Xinminqingma & Guizhou \\
\hline 2 & Ganzaerhao & Jiangxi & 56 & Youqima & Hunan \\
\hline 3 & Miaobazhuma & Sichuan & 57 & Baiyema & Jiangxi \\
\hline 4 & Simaohongzhuma & Yunnan & 58 & Lichuanhoupizhuma & Jiangxi \\
\hline 5 & Shuiqingzhuma & Chongqing & 59 & Boyanghuangyema & Jiangxi \\
\hline 6 & Yichuntongpiqing & Jiangxi & 60 & Xiaoqinggan & Sichuan \\
\hline 7 & Yachibaima & Guizhou & 61 & Yihuangjiama & Jiangxi \\
\hline 8 & Rongchangzhuma & Chongqing & 62 & Xiangningdayelv & Hubei \\
\hline 9 & Guangdonghuangpidouerhao & Guangdong & 63 & Yedouzi & Jiangxi \\
\hline 10 & Qingyezhuma & & 64 & Tiantaitiema & Zhejiang \\
\hline 11 & Hexianjiama & Guangxi & 65 & Dayehongzhameng & Jiangxi \\
\hline 12 & Guangpimayihao & Jiangxi & 66 & Jiangkouqingpizhuma & Guizhou \\
\hline 13 & Juandongtuma & Chongqing & 67 & Longtangbaima & Chongqing \\
\hline 14 & Limuqingma & Guizhou & 68 & Wuchangshanpozhumayihao & Hubei \\
\hline 15 & Dadaoma & Guizhou & 69 & Jinpingqingma & Guizhou \\
\hline 16 & Lipingqingma & Guizhou & 70 & Qingpigan & Jiangxi \\
\hline 17 & Lidazhuma & Yunnan & 71 & Fenyihuangguangdou & Jiangxi \\
\hline 18 & Gebuqingma & Guangxi & 72 & Huangjiuma & Hunan \\
\hline 19 & Tongmuqingma & Guangxi & 73 & Guangpimaerhao & Jiangxi \\
\hline 20 & Qingpidamayihao & Chongqing & 74 & Qianzhuyihao & Guizhou \\
\hline 21 & Gaoanma & Jiangxi & 75 & Loushanhuangpima & Guizhou \\
\hline 22 & Yujiangma & Jiangxi & 76 & Anlongzhumaerhao & Sichuan \\
\hline 23 & Fulisima & Chongqing & 77 & Linggongqingpima & Guizhou \\
\hline 24 & Wuchuanbaima & Guizhou & 78 & Japanzhumaqihao & Japan \\
\hline 25 & Huanhancongma & Sichuan & 79 & Xieliqingma & Chongqing \\
\hline 26 & Nanchongzhuma & Sichuan & 80 & Kuguaqing & Hunan \\
\hline 27 & Xiningxianma & Chongqing & 81 & Longhuibaimayihao & Hunan \\
\hline 28 & Puqidayelvyihao & Hubei & 82 & Yongshanzhuma & Yunnan \\
\hline 29 & Wulonghonggan & Chongqing & 83 & Honggujin & Hubei \\
\hline 30 & Xiaogubai & Jiangxi & 84 & Yushanma & Jiangxi \\
\hline 31 & Rongjiangbaimayihao & Guizhou & 85 & Yinniyihao & Indonesian \\
\hline 32 & Niutima & Hunan & 86 & Sichuangaodibaima & Chongqing \\
\hline 33 & Nanchenghoupizhuma & Jiangxi & 87 & Yinnierhao & Indonesian \\
\hline 34 & Huangqingdou & Jiangxi & 88 & Shanqingbaima & Chongqing \\
\hline 35 & Zixima & Jiangxi & 89 & Ershiqingmaerhao & Hubei \\
\hline 36 & Datianhuangganzhuma & Yunnan & 90 & Xinningqingma & Hunan \\
\hline 37 & Ningduyema & Jiangxi & 91 & Ximatuma & Guangxi \\
\hline 38 & Heipima & Guangxi & 92 & Yinnisanhao & Indonesian \\
\hline 39 & Tianbaomayihao & Jiangxi & 93 & Zunyichuangenma & Guizhou \\
\hline 40 & Changshaqingyema & Hunan & 94 & Dayujiandaobai & Hubei \\
\hline 41 & Chuanzhuerhao & Sichuan & 95 & Xiaoyelugan & Jiangxi \\
\hline 42 & Nanchengbaopizhuma & Jiangxi & 96 & Pingchangjiama & Sichuan \\
\hline 43 & Ningduqingzhuma & Jiangxi & 97 & Leiyanghuangkema & Hunan \\
\hline 44 & Manqiangzhuma & Hubei & 98 & Gaodiqingma & Chongqing \\
\hline 45 & Lvzhubai & Jiangxi & 99 & Dingyezhuma & Guangxi \\
\hline 46 & Dayujiandaobai & Hubei & 100 & Kanlazhuma & Myanmar \\
\hline 47 & Quxianzhuma & Zhejiang & 101 & Shengbaxianma & Hubei \\
\hline 48 & Zhuzibian & Jiangxi & 102 & Xinpuqingma & Guizhou \\
\hline 49 & Yangshuojigubai & Guangxi & 103 & Tianpaishanyema & Guangxi \\
\hline 50 & Hupima & Jiangxi & 104 & Huangjindou & Hubei \\
\hline 51 & Yangxinxiyelv & Hubei & 105 & Chongrenzhuma & Hunan \\
\hline 52 & Xiangzhuliuhao & Hunan & 106 & Gegenma & Hunan \\
\hline 53 & Ningdudabaima & Jiangxi & 107 & Tianbaomaerhao & Jiangxi \\
\hline 54 & Xiangzhuyihao & Hunan & 108 & Huangpinghuangganma & Guizhou \\
\hline
\end{tabular}




\section{SSR primers} et al. (2011).

Twenty-one SSR primer combinations (Table 2) were synthesized according to Chen

Table 2. Primer sequence of simple sequence repeat (SSR) used among ramie accessions.

\begin{tabular}{lll}
\hline Locus & Primer sequences $\left(5^{\prime}-3^{\prime}\right)$ & Primer sequences $\left(5^{\prime}-3^{\prime}\right)$ \\
\hline b50 & F: AACAATCCAGGAGTGGCAATC & R: ACAAGCGAAGATCGTCTCATC \\
b35 & F: CGTTCAGTCACCAGCAAGG & R: GAGGGAAGCAGGGAGAGC \\
b38 & F: TAATCCCTCAATGGCTCTTTC & R: GAGAAGGATACGAATTGACAGG \\
b40 & F: TGTATAGAACTGAGTAAATGATTG & R: CAACTTTCTTAAACCACTTTCG \\
b43 & F: CGAGCCTTCTTCTTCTTCTGG & R: GCAAGCAATACGGACAGTAGG \\
c03 & F: CGTGAAAATAGTGATATGTGTG & R: ACTGTAACAATCAAGAAGAAACC \\
c07 & F: GCCACAGCCGAGGAAGAG & R: TCTCATCACCACCACCTTAGG \\
b27 & F: AGCCAGGTTCCAGAAGTCC & R: CATAATCACAAAGTCTCGGTTCC \\
b28 & F: TCCCACCACGGACTACTG & R: AACCACCATCATCATCATCATC \\
b11 & F: GCGGAGGCTTAATTTGCTTTG & R: ACTCAATACATACACGGCACTAG \\
b16 & F: ACCTCTACGGACCTCTTCTTC & R: CATAACATAACATGACACACAAGC \\
b24 & F: GAGCCAGAGCCAGGTTCC & R: ACAAAGTCTCGGTTCCTTACAC \\
b34 & F: AATAGAATGTGGAGGCGATAGAG $:$ AAACCATAAATCAACTACCGAACC \\
b64 & F: CTTGAGATACAGCCTTCCATTAG & R: CACACCTCGCTTCCCTTG \\
c17 & F:GAAACTATTTCCACCAACAAAG & R: ACACACATTCCTACACACC \\
b57 & F: CGGATATGGTGGAGGTTATGC & R: CAGAACGACGACGACGAC \\
b65 & F: ACGAACCACAACACAGAGAG & R: ACGAGGGAACACCAGAGAG \\
c18 & F: AAGCCGAGCGTGAAGAAG & R: ACACACAGAAAGAACACAAGAC \\
b53 & F: GGCTCAAGTTTGCTCATAGATTC & R: CGGCTTCGCTTTAGGATTTG \\
b56 & F: CGGTCTGTGGATACGAATGG & R: GACGACGACGACGATGATG \\
c10 & F: AGTGCGGAGATAACTGTTC & R: GGCTACTTTATTCTAAACCAAAC \\
\hline
\end{tabular}

\section{SSR analysis}

SSR-polymerase chain reactions (PCRs) were carried out in $10-\mu \mathrm{L}$ reaction volumes with 1X PCR buffer, $0.2 \mathrm{mM}$ dNTPs, 1 U Taq DNA polymerase (Tiangen, Beijing, China), $0.5 \mu \mathrm{L}$ forward primer ( $10 \mathrm{nM}$, Tiangen), $0.5 \mu \mathrm{L}$ reverse primer ( $10 \mathrm{~nm}$, Tiangen), and $0.5 \mu \mathrm{L}$ DNA of each accession under the following PCR conditions: $5 \mathrm{~min}$ at $94^{\circ} \mathrm{C}$, followed by 30 cycles for $30 \mathrm{~s}$ at $95^{\circ} \mathrm{C}, 30 \mathrm{~s}$ at the primer-specific annealing temperature, $30 \mathrm{~s}$ at $72^{\circ} \mathrm{C}$, and final extension for $10 \mathrm{~min}$ at $72^{\circ} \mathrm{C}$.

PCR products were separated on $8 \%$ polyacrylamide gels, and silver staining was conducted according to the method described by Zhang et al. (2000). Molecular weights were estimated using a DNA marker (DNA Marker 2000, BioTeke Co., Beijing, China). SSR analysis was repeated at least twice. Clear bands from PCR products were recorded types 1-8. Faint bands were recorded as 0 .

\section{Diversity index (DI) of SSR primers and CHEN specialty index (CHEN-SI) of germplasms}

The diversity index of SSR primers and CHEN specialty index (CHEN-SI) of germplasms were calculated using Genetics Statistics 3.0 (2007SR11872) developed by North-East Agriculture University, China.

Simpson's DI (Simpson):

$$
\mathrm{D}=\dot{1}-\Sigma(\operatorname{Pi} 2)
$$

(Equation 1) 
In the formula, Pi was the proportion of band types of a locus.

Simpson's unbiased DI (unbiased Simpson):

$$
\mathrm{D}=\overline{1}-\Sigma((\mathrm{ni} \times(\mathrm{ni}-1)(\mathrm{N} \times(\mathrm{N}-1)))
$$

(Equation 2)

In the formula, ni was the number of band types in a locus; $\mathrm{N}$ was the total number of all band types counted in a locus.

Shannon-Weiner's DI (Shannon-Weiner):

$$
\mathrm{H}^{\prime}=-\Sigma(\mathrm{Pi} \times \log (\mathrm{Pi}))
$$

In the formula, Pi was the proportion of band types in a locus.

Brillouin's DI (Brillouin):

$$
\mathrm{H}=(1 / \mathrm{N}) \times \log (\mathrm{N} ! / \mathrm{n} 1 \ln 2 \ln 3 ! \ldots \mathrm{ns} !)
$$

(Equation 4)

In the formula, $\mathrm{n} 1$ !n 2 !n $3 ! \ldots n s$ ! was the factorial product of each band type in a locus. CHEN specialty index (CHEN-SI):

$$
\mathrm{CHEN}-\overline{\mathrm{SI}}=(\dot{\Sigma}(1 /(\mathrm{Nij} / \mathrm{Mj})))(\mathrm{m} \times \mathrm{n})(\mathrm{i}=1,2,3 \ldots \mathrm{m} ; \mathrm{j}=1,2,3 \ldots \mathrm{n}) \quad \text { (Equation } 5)
$$

In the formula, Nij was the total distributive number of band type in the $\mathrm{m}$ individual of the $\mathrm{i}^{\text {th }}$ individual in the $\mathrm{j}$ locus; $\mathrm{Mj}$ is the total number of band type of the $\mathrm{j}$ locus, its number is $\mathrm{m}$ in the data of dominant molecular marker.

The Chi-square analysis was used to analyze the data. $\mathrm{P} \leq 0.01$ indicated extreme significance; $\mathrm{P} \leq 0.05$ indicated significance; and $\mathrm{P}>0.05$ indicated no significance.

\section{ID construction}

The molecular ID of ramie germplasms was constructed using ID Analysis 3.0 (Northeast Agriculture University, China).

\section{RESULTS}

\section{Band type developed by SSR primers}

The number of alleles per locus ranged from 2 to 3 among the 21 primer pairs. Fourteen primers amplified 2 alleles, while 7 primers amplified 3 alleles.

The number of band types developed by SSR primers was 2-9. Of the 21 primers, b50 amplified the largest number of band types (9 band types), while b64 and c17 amplified the smallest number of band types ( 2 band types).

\section{DI of SSR primers}

The DI of SSR primers is listed in Table 3, including 4 type DI consisting of Simpson's diversity index, Simpson's unbiased diversity index, Shannon-Weiner's diversity index, 
and Brillouin's diversity index. Values ranged from 0.158 to $0.808,0.159$ to $0.816,0.146$ to 0.747 , and 0.133 to 0.702 , respectively. Among the 21 SSR primers, primer c10 showed the highest DI and primer $\mathrm{c} 07$ showed the lowest DI.

\begin{tabular}{|c|c|c|c|c|c|}
\hline SSR primers & Band type & Simpson & US & SW & $\mathrm{BR}$ \\
\hline b50 & 9 & 0.751 & 0.758 & 0.663 & 0.619 \\
\hline b35 & 5 & 0.701 & 0.708 & 0.55 & 0.521 \\
\hline b38 & 4 & 0.527 & 0.532 & 0.349 & 0.332 \\
\hline b40 & 7 & 0.664 & 0.67 & 0.569 & 0.532 \\
\hline b43 & 3 & 0.477 & 0.481 & 0.291 & 0.281 \\
\hline $\mathrm{c} 03$ & 5 & 0.688 & 0.694 & 0.542 & 0.514 \\
\hline c07 & 4 & 0.158 & 0.159 & 0.146 & 0.133 \\
\hline b27 & 4 & 0.614 & 0.62 & 0.44 & 0.421 \\
\hline b28 & 6 & 0.619 & 0.626 & 0.487 & 0.455 \\
\hline b11 & 4 & 0.664 & 0.67 & 0.475 & 0.456 \\
\hline b16 & 4 & 0.602 & 0.608 & 0.428 & 0.408 \\
\hline b24 & 4 & 0.641 & 0.647 & 0.46 & 0.44 \\
\hline b34 & 5 & 0.587 & 0.592 & 0.455 & 0.43 \\
\hline b64 & 2 & 0.356 & 0.359 & 0.235 & 0.225 \\
\hline c17 & 2 & 0.486 & 0.491 & 0.295 & 0.285 \\
\hline b57 & 4 & 0.672 & 0.678 & 0.543 & 0.516 \\
\hline b65 & 7 & 0.617 & 0.623 & 0.522 & 0.488 \\
\hline c18 & 8 & 0.735 & 0.743 & 0.701 & 0.65 \\
\hline b53 & 7 & 0.709 & 0.715 & 0.636 & 0.595 \\
\hline b56 & 8 & 0.783 & 0.791 & 0.715 & 0.666 \\
\hline c10 & 7 & 0.808 & 0.816 & 0.747 & 0.702 \\
\hline
\end{tabular}

Simpson indicated Simpson's diversity index; US indicated Simpson's unbiased diversity index; SW indicated Shannon-Weiner's diversity index; BR indicated Brillouin's diversity index.

\section{Special band type}

Two primers amplified special band types. The 5 th special band type developed by primer b50 could distinguish Lipingqinma among 108 ramie germplasms. The 1st and 5th special band types developed by primer b65 could distinguish Ganzaerhao and Shanqingbaima, respectively.

\section{CHEN-SI of germplasm}

The CHEN-SI values of 108 germplasms are listed in Table 4 . There were significant differences $(\mathrm{P}<0.01)$ among the $\mathrm{CHEN}-\mathrm{SI}$ of the 108 ramie germplasms, ranging from 44.082 to 218.163. There were 2 germplasms with CHEN-SI values greater than 200 , including the 88th with 218.163 and the 16th with 201.988. There were 4 germplasms showing CHEN-SI values less than 50, including the 68th with 49.825, the 69th with 48.216, the 70th with 45.86, and the 74th with 44.082. The high CHEN-SI indicates that ramie germplasms have a large number of special band types, and thus the results may be useful for identifying and preserving germplasm resources of ramie.

\section{Germplasm ID construction}

The PCR amplification banding pattern of 108 ramie germplasms was recorded, which was amplified by 21 SSR primer combinations, as well as being expressed $1,2 \ldots \mathrm{n}$, respectively. Next, the molecular identity card of ramie was constructed according to the following 
M.B. Luan et al.

Table 4. CHEN index and ID of germplasms.

\begin{tabular}{|c|c|c|c|c|c|}
\hline Germplasm code & CHEN index & ID & Germplasm code & CHEN index & ID \\
\hline 1 & 79.267 & 43311132 & 55 & 99.914 & 17646213 \\
\hline 2 & 176.671 & 23122223 & 56 & 95.824 & 12326313 \\
\hline 3 & 74.214 & 23413323 & 57 & 69.994 & 74323113 \\
\hline 4 & 52.968 & 24434323 & 58 & 153.699 & 35326332 \\
\hline 5 & 71.503 & 12435223 & 59 & 101.934 & 47640132 \\
\hline 6 & 56.267 & 24221233 & 60 & 136.545 & 11324132 \\
\hline 7 & 58.93 & 54326123 & 61 & 67.944 & 43325322 \\
\hline 8 & 96.198 & 25221113 & 62 & 97.201 & 74626233 \\
\hline 9 & 92.772 & 42444113 & 63 & 59.451 & 44235332 \\
\hline 10 & 85.013 & 45425323 & 64 & 82.951 & 32211132 \\
\hline 11 & 98.284 & 37214303 & 65 & 98.17 & 34415312 \\
\hline 12 & 92.397 & 54215323 & 66 & 95.376 & 30265122 \\
\hline 13 & 107.657 & 27221113 & 67 & 59.35 & 30244122 \\
\hline 14 & 73.91 & 24336333 & 68 & 49.825 & 74236133 \\
\hline 15 & 98.006 & 32227223 & 69 & 48.216 & 50236133 \\
\hline 16 & 201.988 & 32453122 & 70 & 45.86 & 44224123 \\
\hline 17 & 68.989 & 34436223 & 71 & 75.213 & 34231122 \\
\hline 18 & 79.796 & 25248123 & 72 & 80.116 & 17221222 \\
\hline 19 & 72.202 & 24223213 & 73 & 96.707 & 14446232 \\
\hline 20 & 81.511 & 24221123 & 74 & 44.082 & 40256033 \\
\hline 21 & 57.924 & 22334133 & 75 & 61.489 & 54233133 \\
\hline 22 & 98.259 & 24321233 & 76 & 59.744 & 44233032 \\
\hline 23 & 87.989 & 13224123 & 77 & 68.141 & 25221133 \\
\hline 24 & 89.338 & 55201333 & 78 & 72.132 & 54220233 \\
\hline 25 & 59.163 & 34323323 & 79 & 89.832 & 72334332 \\
\hline 26 & 80.615 & 35329322 & 80 & 81.766 & 44424122 \\
\hline 27 & 92.963 & 45221232 & 81 & 96.743 & 4465123 \\
\hline 28 & 85.17 & 24625223 & 82 & 85.892 & 77333223 \\
\hline 29 & 123.05 & 37225222 & 83 & 85.563 & 37621333 \\
\hline 30 & 71.814 & 54221133 & 84 & 57.149 & 44225132 \\
\hline 31 & 68.646 & 43226223 & 85 & 59.287 & 20225132 \\
\hline 32 & 57.626 & 34233323 & 86 & 110.208 & 72334222 \\
\hline 33 & 59.482 & 54221333 & 87 & 55.507 & 2225232 \\
\hline 34 & 68.98 & 27226323 & 88 & 218.163 & 21541132 \\
\hline 35 & 76.586 & 53225323 & 89 & 89.272 & 23424323 \\
\hline 36 & 130.457 & 27235323 & 90 & 58.964 & 54224222 \\
\hline 37 & 58.597 & 7226123 & 91 & 84.068 & 72430222 \\
\hline 38 & 62.598 & 37234123 & 92 & 66.301 & 72237322 \\
\hline 39 & 110.406 & 54215123 & 93 & 96.842 & 27637332 \\
\hline 40 & 81.42 & 43224332 & 94 & 84.532 & 54615322 \\
\hline 41 & 108.933 & 24265323 & 95 & 73.37 & 77225223 \\
\hline 42 & 70.83 & 72225113 & 96 & 68.476 & 34225212 \\
\hline 43 & 71.802 & 52234112 & 97 & 61.951 & 24225232 \\
\hline 44 & 67.845 & 32249322 & 98 & 74.089 & 14243222 \\
\hline 45 & 96.457 & 44424323 & 99 & 67.123 & 22234132 \\
\hline 46 & 97.761 & 34231123 & 100 & 62.668 & 47321322 \\
\hline 47 & 84.048 & 34224223 & 101 & 80.758 & 25313123 \\
\hline 48 & 75.369 & 44214103 & 102 & 67.52 & 74325232 \\
\hline 49 & 83.857 & 23217312 & 103 & 71.901 & 14234233 \\
\hline 50 & 69.219 & 24221312 & 104 & 62.803 & 54334132 \\
\hline 51 & 74.177 & 44426312 & 105 & 69.151 & 34432323 \\
\hline 52 & 108.04 & 23418313 & 106 & 82.076 & 24325222 \\
\hline 53 & 66.518 & 24231213 & 107 & 81.69 & 22333332 \\
\hline 54 & 95.737 & 14246213 & 108 & 157.241 & 57224333 \\
\hline
\end{tabular}

steps. First, we deleted SSR primers when the proportion of blurred amplification belt was above $4 \%$. Six SSR primer combinations were deleted, including b35, c03, b28, b16, c18, and b56. The second step was to delete SSR primers when the similarity coefficient was greater than 0.8 with others. Two SSR primers were deleted, including c07 and b24. Finally, we constructed a molecular identity card based on the specificity index of SSR primers. The germ- 
plasm resources of ramie were distinguished using the specific allele of more than 2 marker combinations. The distinction would be made by increasing the number of markers until they were not distinguished entirely using 2 markers, i.e. the k-th marker combinations amplified $n$ banding patterns corresponding to the k-th bits of molecular identity. In current study, the 108 germplasm resources of ramie were distinguished using 8 SSR primer combinations $(k=8)$, including $\mathrm{c} 10, \mathrm{~b} 53, \mathrm{~b} 65, \mathrm{~b} 40, \mathrm{~b} 50, \mathrm{~b} 11, \mathrm{~b} 27$, and $\mathrm{c} 17$; the 8-bit molecular identity card (ID) was constructed (Table 4). For instance, the molecular ID of ramie known as Wayaozhuma was 43311132 . The 1 st 4 indicated that the b20 primer (ki) amplified its 4 th banding pattern (nj), and the $3 \mathrm{rd} 3$ indicated that the $\mathrm{b} 65$ primer amplified its $3 \mathrm{rd}$ banding pattern.

\section{DISCUSSION}

The banana fingerprints developed by Wang et al. (2009) can be considered molecular identification, and the authors combined letters with numbers to determine the digits of molecular identification and different alleles amplified by SSR primers. For example, the 11th allele amplified by the first marked primers was referred to as A11, resulting in a large number of string code digits in the molecular identification. Wang et al. (2010) previously constructed ramie molecular identification using 0 or 1 notation including the 16-digit code using ISSR markers. In the present study, the amplification results of the Nth marker's primers directly corresponded to the Nth bit in molecular identification, omitting the expression of a tag name. Additionally, the molecular identification only had 1 digit in each position because the amplified band pattern of each labeled primers did not exceed 9. Compared to the previous ramie molecular identification studies, this method is very simple to record.

With the continuous development of DNA molecular markers and improvement of detection technology, identifying crop variety resources traditionally based on morphological characteristics has become focused on the DNA level. Recently, molecular identity cards have been used for variety characteristic digitization. In this study, we concluded that a string code could be applied to express the molecular identification of crop variety resources and to determine the differences among varieties.

Although appraisal using molecular markers has advantages that morphological appraisal does not, there were some limitations to our study. Choosing concrete molecular markers may influence the analysis because of their respective shortcomings. Our previous study established 42 molecular IDs of ramie using ISSR molecular markers. However, ISSR technology is prone to error and deviations because of the large number of amplified bands and the difficulties in duplication. SSR molecular markers frequently result in high duplication. However, this method is easy and does not require a DNA digestion step. These characteristics make it possible to establish molecular IDs. SSR markers are stable over different generations, according to SSR analysis of parents and their descendants in the study of barley genealogy. The SSR stability of different individuals of the same species has been verified in rice (Akagi et al., 1997). Therefore, SSR marker-based molecular ID is more accurate and reliable compared with ISSR markers.

Although we identified 108 ramie germplasms using 8 pairs of SSR primers in this study, the character differences for each germplasm could not be directly determined because the corresponding relationship between various allele and their corresponding agronomic characters were not identified. Although traditional morphological identification requires a longer period and is greatly affected by environmental factors, it is simple, visible, and easy 
to operate, which is in contrast to molecular markers. Therefore, this method is applicable for identifying ramie germplasm resources, combining traditional morphological identification with molecular marker identification to establish a system for germplasms ID.

Understanding the genetic background of germplasms is vital for breeding. The samples tested in this study were collected from different origins, indicating the wide applicability for breeding. Thus, the molecular identification (ID) of each species can represent specificity. The results showed that molecular ID is a powerful approach for distinguishing germplasm resources regardless of their origin, genetic pedigree, or morphological similarity. For example, the xiangzhu1 and qianzhu1 ramie varieties have similar genetic backgrounds because they have the same same parent, huangkezao, with the molecular IDs 14246213 and 40256033, respectively. Therefore, molecular ID may provide a reference for germplasm identification and variety selection.

For additional ramie germplasms, a larger number of special bands from special germplasms developed using suitable primers can be used to construct the molecular ID in ramie. However, it is possible for ramie germplasms of different varieties to have the same molecular ID because of the limited number of SSR primers available. Thus, it is necessary to expand the number of SSR primers.

\section{ACKNOWLEDGMENTS}

Research supported by the National Natural Science Foundation of China (Youth Program) (Grant \#30900913), Hunan Provincial Natural Science Foundation of China (Grant \#13JJ4116), and Science and Technology Funds Program of CAAS (2009). We specially thank for Dr. Zhenbang Hu of North-east Agricultural University who provided the Genectics 3.0 and IA1.0 software and guidance.

\section{REFERENCES}

Akagi H, Yokozeki Y, Inagaki A and Fujimara T (1997). Highly polymorphic microsatellites of rice consist of AT repeats and a classification of closely related cultivars with these microsatellite loci. Theor. Appl. Genet. 94: 61-67.

Chen CW, Cao K, Wang LR, Zhu GR, et al. (2011a). Molecular ID establishment of main china peach varieties and peach related species. Sci. Agric. Sin. 44: 2081-2093.

Chen JH, Luan MB, Song SF, Zou ZZ, et al. (2011b). Isolation and characterization of EST-SSRs in the ramie. Afr. J. Microbiol. Res. 5: 3504-3508.

Ding MZ, Pan GT, Zhang ZH, Yang Y, et al. (2008). Genetic relation analysis of Boehmeria nivea cultivars or lines from Sichuan with ISSR and construction of the molecular markers linked Tomale sterile gene. J. Nuclear Agric. Sci. 22: 183-187.

Fang XJ, Wu WR and Tang JL (2001). DNA marker assisted selection in crop breeding. Science Press, Beijing.

Gao YL, Zhu RS, Liu CY, Fu LW, et al. (2009). Establishment of molecular ID in soybean varieties in Heilongjiang, China. Acta Agron. Sin. 35: 211-218.

Guo AP, Zhou P, Peng S and Zheng X (2001). Factors affecting the reaction system of RAPD analysis in Boehmeria Jacq. Chin. J. Trop. Crops 22: 64-69.

Guo Y, Xiong HP, Chen P, Wang YZ, et al. (2013). Analysis on genetic diversity of S1 progeny of ramie variety Zhongzhu No. 2 by SSR markers. Plant Fiber Sci. China 35: 75-80.

Hou SM, Duan JQ, Liang XN, Ding XW, et al. (2006). Detection for mtDNA of cytoplasmic male sterile (CMS) line and maintainer line of ramie [Boehmeria nivea (L.) Gaud.] by ISSR. Plant Physiol. Commun. 42: 705-707.

Jie YC, Zhou QW and Chen PD (1999). Genetic relation analysis of ramie genetypes with RAPD marker. China Fiber Crops 21: 1-6.

Liu FH (2002). The ramie germplasms. China Agricultural Science and Technology Press, Beijing. 
Liu LJ, Sun ZX and Peng DX (2006). Optimization for ISSR reaction system in ramie (Boehmeria nivea L. Gaud.). Chin. Agric. Sci. Bull. 22: 64-68.

Liu LJ, Peng DX and Wang B (2008). Genetic relation analysis on ramie (Boehmeria nivea L. Gaud.) inbred lines by SRAP markers. Agric. Sci. China 7: 944-949.

Liu Q, Dai ZG, Chen JQ, Wen L, et al. (2013). Establishment of molecular identity for kenaf germplasm using SRAP marker. Sci. Agric. Sin. 46: 1974-1983.

Meng ZQ, Liu LJ, Wang B and Peng DX (2010). Double orthogonal optimization for RAPD-PCR reaction system in Boehmeria Jacq. J. Yunnan Agric. Univ. 25: 758-762.

Tu X and Chen SC (2007). Ramie-an effective plant for soil and water conservation in the southern sloped farmlands. Global Seabuckthorn Res. Dev. 5: 45-48.

Wang JY, Chen YY, Huang BZ, Fei Y, et al. (2009). Establishment of fingerprinting for bananas (Musa nana) by SSR marker. J. Fruit Sci. 26: 733-738.

Wang LM, Jiao SJ, Jiang YX, Yan HD, et al. (2011). Establishment of molecular identity in 142 sweet sorghum varieties. Acta Agric. Sin. 37: 1975-1983.

Wang XF, Chen JH, Luan MB, Qi JM, et al. (2010). Establishment of molecular identification in ramie germplasms. $J$. Plant Genet. Germplasms 11: 802-805.

Wen L, Yu CM, Wang YZ, Chen P, et al. (2011). Application of molecular marker SRAP on analysis of genetic diversity of polyembryonic ramie seedlings. J. Hunan Agric. Univ. (Nat. Sci.) 37: 243-247.

Xiong HP (2008). Bast-fiber crops breeding. China Agricultural Science and Technology Press, Beijing.

Xiong HP (2010). The production status and policy suggestion of bast and leaf fiber crops in China. Plant Fiber Sci. China 32: 301-304.

Yang Y, Liu Z, Zhao Y and Liang GG (2010). Construction of DNA fingerprints for tea cultivars originated from Hunan province. J. Tea Sci. 30: 367-373.

Yu CM, Chen JR, Wang YZ, Zhu AG, et al. (2007). Research progresses of molecular breeding in ramie and forage ramie. Plant Fiber Sci. China 29: 389-392.

Zhang J, Wu YT, Guo WZ and Zhang TZ (2000). Fast screening of microsatellite markers in cotton with page/silver staining. Acta Gossypii Sin. 12: 267-269.

Zhang JG, Tian R, Chen QL, Yang XP, et al. (2014). Establishment of molecular ID for pear cultivars based on SSR markers. J. Huazhong Agric. Univ. 33: 12-17.

Zheng HY, Su JG, Dai ZG, Li Y, et al. (2010). Establishment of molecular identity for kenaf germplasm using ISSR and RAPD markers. Sci. Agric. Sin. 43: 3499-3510.

Zhou JL, Jie YC and Jiang YB (2004). Genetic relation analysis on ramie cultivars with microsatellite markers. Acta Agron. Sin. 30: 289-292.

Zou ZZ, Chen JH, Luan MB, et al. (2012). Evaluation of genetic relationship in ramie based on RSAP, SRAP, and SSR. Acta Agron. Sin. 38: 840-847. 